Editorial

\title{
Single atom modeling resolution in drug design for therapy
}

Keywords: pharma, fortunes, acquisition, medicine, technology, composing domains

\section{Editorial}

Drug design is one of the more fascinating, difficult and rewarding activities in applied sciences to medicine:

Richness and power of big Pharma are essentially based on it, as well as start-up success. A single good molecule could make fortunes, nowadays as it did! Not surprisingly, it is thus quite searched for, both in big Pharma and in small start-up hoping to make the seed for a sudden growth or acquisition.

As most of design activities, it is a technology based on science but much resorting to art: if it would be straightforward, everybody would be able to, thus no big deal would be anymore when discovered!

Traditionally based on intuition, then supported by "wet" trial and errors, it is nowadays more and more supported by simulation tools aiding to forecast in silico the properties of the final molecule form the properties of its composing domains.

A recent pervasive technology, for instance, namely machine learning, is indeed quite useful also in such a sense, for instance at least in forecasting hydrophily versus hydrophoby of the whole molecule, taking into account some properties of the components together with some example of analogous.

Nowadays power parallel computation on one side, together with deeper phisico-chemical understanding on the other side, allow even more: to even simulate the whole molecule by building it atom by atom, thus more practically by substituting a single atom as well as
Volume 2 Issue 5 - 2018

\author{
Diego Liberati \\ Department of Bioengineering, Polytechnic University of Milan, \\ Italy
}

Correspondence: Department of Bioengineering, Polytechnic University of Milan, Piazza Leonardo da Vinci 32, 20133 Milano, Italy, Tel +3934805693 I7, Email diego.liberati@polimi.it

Received: August 28, 2018 | Published: September 05, 2018

a few atoms or more typically a single domain with respect to an already known molecule whose properties are almost, or at least some of, the desired ones.

Such capability opens an immense spectrum of possibilities, whose management will more and more require people, or at least coordinate teams, integrating solid phisico-chemical skills to drug effect expectation experience, thus pushing even further to transdisciplinary continuous education also in such fascinating frontier field.

\section{Acknowledgments}

None.

\section{Conflict of interest}

Author declares that there is no conflict of interest. 